\title{
Autorregulación y empresas ciudadanas: cartilla de lineamientos legales para emprendedores que trabajan con nanotecnología
}

\section{Self-regulation and citizen companies: the booklet on law guidelines for entrepreneurs that work with nanotechnology}

\author{
Fernanda Felitti da Silva D'avila,*, Daniele Weber S. Leal," Wilson Engellman* \\ Raquel von Hohendorff*
}

\begin{abstract}
Currently there is a growth in the use of nanotechnologies, within the fourth industrial revolution, providing a series of concerns in relation to ethical, legal and social aspects, as well as risks to health and the environment. Given this reality, with many risks, it has become increasingly difficult for the law and the State to follow up on the production of legislation. We seek to answer, with this article, the following research question, from a systemic-constructivist methodological perspective: is it possible to create regulatory legal scenarios based on the different legal principles existing in Brazilian law, aiming at the supervision of nanotechnologies and nanomaterials, at the light of Gunther Teubner's legal pluralism? For this, the concepts of risk were presented, based on the studies of Luhmann and Ulrich Beck, the ideas of legal pluralism of Gunther Teubner, of citizen companies and of regulated self-regulation. And at the end, an example of this solution was presented, through the Law guidelines booklet for the industrial and commercial segment that works with nanotechnologies. In conclusion, through regulated selfregulation it is possible to create regulatory legal scenarios, which enable legal plurality, to help in normative production, seeking to keep pace with changes in society.

KEYWORDS: nanotechnology, fourth industrial revolution, legal pluralism, regulated self-regulation.
\end{abstract}

RESUMEN: En la actualidad existe un crecimiento en el uso de las nanotecnologías, dentro de la cuarta revolución industrial, generando una serie de preocupaciones en relación con los aspectos éticos, legales y sociales, asi como sobre los riesgos para la salud y el medio ambiente. Ante esta realidad de tantos riesgos, se ha vuelto cada vez más dificil para la ley y el Estado dar seguimiento a la producción de legislación. Con este artículo buscamos responder, desde una perspectiva metodológica sistémico-constructivista, la siguiente pregunta de investigación: ¿es posible crear escenarios legales regulatorios a partir de los diferentes principios legales existentes en el derecho brasileño, apuntando a la supervisión de nanotecnologías y nanomateriales, a la luz del pluralismo jurídico de Gunther Teubner? Para ello, nos basamos en los conceptos de riesgo, a partir de los estudios de Luhmann y Ulrich Beck, la idea de pluralismo jurídico de Gunther Teubner, de empresa ciudadana y de autorregulación regulada. Al final, se presenta un ejemplo de esta solución, a través de la Cartilla de lineamientos legales para el segmento

Recibido: 29 de mayo, 2021. Aceptado: 27 de julio, 2021. Publicado: 17 de septiembre, 2021.

${ }^{*}$ Universidade do Vale do Rio dos Sinos (UNISINOS), Brasil.

- Autora de correspondencia: fernandafelitti@gmail.com 
industrial y comercial que trabaja con nanotecnologías. En conclusión, a través de la autorregulación regulada es posible crear escenarios legales regulatorios que posibiliten la pluralidad jurídica para ayudar en la producción normativa, buscando seguir el ritmo de los cambios en la sociedad.

PALABRAS CLAVE: nanotecnología, cuarta revolución industrial, pluralismo jurídico, autorregulación regulada.

\section{Introducción}

El uso de las nanotecnologías en el mundo está aumentando cada vez más, haciendo de esta tecnología una de las principales novedades del sector productivo. Esta ascensión de la nanotecnología, enmarcada en el contexto de la cuarta revolución industrial explicado por Klaus Schwab, plantea una serie de preocupaciones sobre aspectos éticos, legales y sociales, así como sobre los riesgos para la salud y el medio ambiente -dado que las mismas características que hacen que los nanomateriales sean diferentes y beneficiosos, también generan dudas y preocupaciones sobre su comportamiento, especialmente en cuanto a su interacción con el ecosistema-. Así, ante la existencia de varios riesgos potenciales en el uso de esta tecnología, ya sea en relación con la salud humana o con el medio ambiente, buscamos, con este artículo, dar respuesta a la siguiente pregunta de investigación, desde la perspectiva metodológica sistémico-constructivista: ¿es posible crear escenarios legales regulatorios basados en los diferentes principios legales existentes en el derecho brasileño, con el objetivo de supervisar las nanotecnologías y los nanomateriales, a la luz del pluralismo jurídico de Gunther Teubner?

A partir de esta pregunta de investigación, en la primera sección de este trabajo se busca comprender el contexto de la cuarta revolución industrial en el que se inserta hoy la nanotecnología, así como analizar la cuestión de los riesgos de esta tecnología, a la luz de la teoría sociológica del riesgo de sus autores principales Niklas Luhmann y Ulrich Beck. Posteriormente, se explica la visión de Teubner sobre el pluralismo jurídico en relación con la falta de legislación compatible con el avance de la nanotecnología.

En la segunda parte del artículo se presentan los conceptos de seguridad desde el diseño (safe by design), RRI (responsible research and innovation), ELSA (ethical, legal and social aspects) y empresa ciudadana, que son el baluarte de una solución para la pregunta de investigación propuesta. A partir de estas herramientas podrá ser comprendida y aplicada la autorregulación regulada.

Como ejemplo de la aplicación de estas herramientas, en el último punto del artículo, se presenta la cartilla de lineamientos legales para el segmento industrial y comercial que trabaja con nanotecnología, una iniciativa del Grupo de Investigación JusNano de la Universidade do Vale do Rio dos Sinos (UNISINOS/Brasil), que busca contribuir a la creación de una regulación de la nanotecnología, con el uso de información de los diferentes principios legales existentes, en el derecho brasileño, a los empresarios que trabajan con nanotecnologías y nanomateriales. 
En conclusión, es a través de este tipo de producción científica y normativa que se puede llegar a una solución para el problema propuesto, la autorregulación regulada, herramienta que permite regular las nuevas tecnologías en el contexto de la cuarta revolución industrial, a través del derecho, cumpliendo su rol protector de los derechos humanos, el derecho al medio ambiente y el derecho al desarrollo sostenible.

\section{La nanotecnología, sus riesgos y la cuarta revolución industrial}

La cuarta revolución industrial, es un concepto acuñado por Klaus Schwab (2016) para explicar un movimiento en marcha desde el cambio de siglo, relacionado con el impacto y las consecuencias de las innovaciones tecnológicas disruptivas como la inteligencia artificial, la robótica, la Internet, los vehículos autónomos, las impresiones en 3D, la biotecnología, el almacenamiento de energía, la computación cuántica y las nanotecnologías.

Schwab explica que lo que diferencia a la cuarta revolución industrial de las tres revoluciones tecnológicas anteriores es la velocidad, amplitud y profundidad, además de la fusión de tecnologías y la interacción entre los dominios físico, digital y biológico que ocurren (Schwab, 2016). En cuanto a la velocidad, evoluciona a un ritmo exponencial y no lineal, resultado del mundo multifacético y profundamente interconectado en el que vivimos. En relación con la amplitud y profundidad, estas se basan en la revolución digital y combinan varias tecnologías, generando un cambio de paradigma sin precedentes no solo en la economía, sino también en la sociedad y las personas.

Importante resaltar el impacto sistémico de estas tecnologías, el cual implica la transformación de sistemas enteros entre países y dentro de ellos como en organizaciones, industrias y en toda la sociedad (Schwab, 2016) - . Es en este contexto que se insertan las nanotecnologías: conjunto de acciones de investigación, desarrollo e innovación, obtenidas gracias a las propiedades especiales de la materia organizadas a partir de estructuras de dimensiones nanométricas. El término nanotecnología deriva del prefijo griego nános, que significa enano, techne que equivale a artesanía y logos que expresa conocimiento.

Actualmente, la tecnología basada en la nanoescala trae consigo muchas incertidumbres, especialmente en lo que respecta a los riesgos altamente nocivos para la salud y el medio ambiente (Durán, Mattoso y Morais, 2006; Kühnel et al., 2014; Pavlicek et al., 2021). Por lo tanto, se necesita indagar sobre la forma en cómo esta tecnología presenta riesgos para la sociedad, cuáles son estos riesgos y cómo es posible enfrentarlos.

Desde la sociedad industrial hasta la actualidad, la sociedad ha adquirido diferentes denominaciones como sociedad de la información, sociedad del riesgo, posmodernidad, modernidad reflexiva, entre otras; su complejidad se ha convertido en hipercomplejidad y el exceso de posibilidades indudablemente condujo a una amplia gama de riesgos y peligros. 
Los riesgos en materia de nanotecnología se encuentran en la combinación de la probabilidad de exposición y los efectos adversos que puedan generarse en este cruce. Es decir, existe la posibilidad de que surjan adversidades en la salud humana, la calidad de vida o la calidad ambiental (Hansen, Baun y Alstrup-Jensen, 2011). En la sociedad moderna, hay más seguridad y mayor riesgo, mayor grado de racionalidad $y$, en conjunto, mayor grado de incalculabilidad de los hechos (Rocha y Martini, 2016).

Ulrich Beck (2003) explica que el mundo de la incertidumbre no cuantificable, creado por nosotros mismos, puede expandirse mucho, siguiendo el ritmo del desarrollo tecnológico. Por lo tanto, las decisiones tomadas en el pasado, con respecto a la energía nuclear, y las actuales, como sobre la ingeniería y exploración de la ingeniería genética, la nanotecnología, la tecnología de la información, por mencionar algunas, son un detonante de consecuencias impredecibles, incontrolables e incluso incomunicables, que amenazan la vida en nuestro planeta.

Desde esta perspectiva, Beck (2010) rescata la importancia de que el conocimiento científico pueda identificar y demostrar que las consecuencias y peligros de la producción industrial desarrollada ahora serían globales, requiriendo que las políticas sean formuladas por instituciones transnacionales. Pero la perspectiva constructivista sería clave para responder a preguntas sobre cómo, por ejemplo, se produce la autoevidencia según qué riesgos son reales, y sobre qué actores, instituciones, estrategias y recursos son determinantes para su fabricación. Es decir, los riesgos existen y no son meramente una construcción social, sino que su transformación depende de cómo se perciban socialmente (Guivant, 2001).

A pesar de los posibles beneficios que trae esta nueva escala de producción, incurrimos en graves riesgos sobre sus efectos sobre el medio ambiente, entrando así en el concepto de sociedad del riesgo (Beck, 2010). Debido a esta preocupación, la difusión de la discusión sobre nanotecnología se ha desarrollado cada vez más a nivel mundial. En relación con este aspecto, se plantea la relevante teoría desenvuelta por el citado autor alemán, sociedad del riesgo, quien, al inicio de su obra Un mundo en riesgo (2003), cuestiona qué tienen en común hechos tan diversos como el desastre de Chernobyl, la alteración del clima, el debate sobre la manipulación genética, la crisis financiera de los países asiáticos y la amenaza actual de ataques terroristas. Enseguida, revela que cualquier discrepancia entre el lenguaje y la realidad es lo que él llama: sociedad mundial del riesgo.

El trabajo de Beck, La sociedad del riesgo mundial: en busca de la seguridad perdida, de 2007 (Beck, 2015), se inspiró en la obra de Luhmann, Sociología del riesgo. Reanudando las discusiones sobre la nueva complejidad presentada en la sociedad, con una percepción globalizada de los riesgos, derivado de las tecnologías; Beck aborda especialmente temas relacionados con daños futuros, dadas las incertidumbres existentes, recordando que las decisiones del presente se reflejarán en el futuro. 
Para Luhmann (2006), la distinción entre riesgo y peligro significa que existe incertidumbre sobre los daños futuros. Se puede considerar que el potencial es consecuencia de la decisión, y entonces hablamos de riesgo y, más precisamente, del riesgo de la decisión. O se entiende que el daño potencial se produce de manera externa, es decir, se le atribuye al medio ambiente, $y$, en este caso, hablamos de peligro. De esta manera, el riesgo se asocia con la decisión, la expectativa, la probabilidad de que ocurran cosas en el futuro, es una comunicación dirigida al futuro.

Luhmann y Beck, dos teóricos de la sociedad del riesgo, convergen en este punto: mientras que el peligro de alguna manera proviene del exterior, el riesgo es un producto derivado, un efecto perverso o secundario (en el sentido de los efectos secundarios indeseables de los fármacos) de nuestras propias acciones.

Así, los riesgos nanométricos son descritos por Carvalho y Damacena (2013) como desastres tecnológicos, siendo parte de los desastres que resultan de la acción humana (antropogénicos). En cualquier caso, los riesgos derivados de las nanotecnologías tienen las características inherentes a la sociedad del riesgo. Se trata de riesgos invisibles, globales, trans-temporales, retardados e irreversibles, pues muestran "un gran potencial de riesgo, por tratarse de una investigación científica que desciende a niveles nunca antes alcanzados" (Engellman, Flores, Weyermüller, 2010: 125).

Por tanto, es necesario cuestionar "la propia prudencia y cautela de la ciencia en el tratamiento de las innovaciones tecnológicas y medioambientales, que, aun trayendo beneficios, están provocando riesgos sociales desmedidos" (Leite y Ayala, 2010: 114). La incertidumbre propia de la sociedad del riesgo resulta imperceptible a la percepción humana y se encuentra, en su mayor parte, en el mundo fisicoquímico, como, por ejemplo, la amenaza nuclear y los elementos tóxicos de los alimentos, conocidos solo a través de información obtenida de estudios científicos. Los riesgos inciertos son característicos del desafío de la nanotecnología, pero son una realidad más allá de la nanotecnología. Abordar la nanotecnología de manera responsable puede ayudar a aprender a lidiar con la amplia gama de riesgos para la salud, la seguridad humana y ambiental en nuestro planeta que cambia rápidamente (Dana, 2012). Sobre estos riesgos, Ost $(1999,345)$ menciona que la situación de incertidumbre reflexiva causada por los riesgos que son simultáneamente globales y transgeneracionales, por tanto, doblemente reflexivos, es producto de nuestras opciones tecnológicas y resultado de nuestros modelos científicos y juicios normativos, reforzando la necesidad de la revisión, basada en la epistemología de la incertidumbre y en la política de la indeterminación.

El riesgo ya no puede verse como un problema del sistema psíquico, conectado al ser humano, sino que es un problema del sistema social, de las comunicaciones de la sociedad que afectan el comportamiento de todos los grupos sociales, variando según las expectativas. Los daños derivados de las decisiones se atribuirán al riesgo; y las desviaciones que surgen del medio ambiente son peligros (Luhmann, 2006). 
Como se mencionó anteriormente, en la idea de Luhmann (2006), lo que define si la situación representa riesgo o peligro es la toma de decisiones. Toda decisión genera algún tipo de riesgo, en mayor o menor grado. Todas las demás adversidades que ocurren y que no surgen de la toma de decisiones, se consideran un peligro. ${ }^{1}$

Así, aunque hoy en día los beneficios de las nanotecnologías dominan nuestros pensamientos cuando hablamos de productos y del mercado, no se debe subestimar el potencial de esta tecnología para causar efectos negativos en la salud humana y el medio ambiente, ya que, debido al tamaño de los materiales (nano), estos no se rigen por leyes de la física clásica — sino de la mecánica cuántica-abriendo posibilidades de que las nanopartículas tengan un mayor grado de toxicidad que en tamaños mayores, es por eso que existe la necesidad de evaluar los riesgos que se derivan de la manipulación, desarrollo y aplicación de estas nuevas tecnologías (Hohendorff, Engellman, 2014).

En noviembre de 2017, la OECD (Organisation for Economic Co-operation and Development) publicó un informe donde analiza una amplia investigación realizada desde 2015 en varios países miembros de la organización, sobre la exposición de los consumidores y el medio ambiente a los nanomateriales manufacturados, mostrando preocupación por los riesgos de estos materiales. El propósito de este informe fue proporcionar un análisis de los resultados del estudio iniciado en 2015, diseñado para recopilar información utilizada para caracterizar las exposiciones de los consumidores y el medio ambiente para la evaluación de riesgos humanos y ecológicos. La investigación estaba destinada a ser utilizada como una herramienta para informar posibles vías de acción y colaboración futuras en el área de evaluación de la exposición en relación con los nanomateriales fabricados (OECD, 2017).

Además de esta importante investigación, en la actualidad hay un aumento de publicaciones e investigaciones sobre los riesgos de las nanopartículas (Kulinowski, en: Foladori, 2015) y este conjunto de publicaciones también debe ser considerado porque representa lo que ya se conoce sobre el comportamiento de algunas partículas cuando se manipulan a escala nanométrica. Hasta 2008, el gobierno federal de Estados Unidos estaba inyectando casi US\$ 60 millones al año en investigación en el área de salud y en los impactos ambientales de la nanotecnología. En 2016, las agencias federales del país propusieron invertir US $\$ 105.4$ millones de dólares en estudios para comprender y abordar los riesgos potenciales de la nanotecnología para el medio ambiente y la salud. Esto representa un aumento masivo del $80 \%$,

${ }^{1}$ La literatura pertinente se encuentra escrita en su inmensa mayoría en inglés. Se dispone en ella de las palabras risk, hazard, danger, pero estas se utilizan, en general, casi en el mismo sentido, Por supuesto, se sabe que en la percepción del riesgo y en su aceptación, la circunstancia tiene un papel importante de que uno se adentre voluntaria o involuntariamente en situaciones de peligro. Y lo mismo es válido en relación con si uno cree tener o no bajo control las consecuencias de la propia conducta" (Luhmann, 2006: 67-68). 
en comparación con los ocho años anteriores, y refleja las preocupaciones actuales sobre lo que no se sabe sobre los riesgos potenciales de los materiales a nano escala diseñados y fabricados expresamente (Maynard, 2016).

Los nanomateriales, como aún tienen gran variabilidad e incertidumbre acerca de su toxicidad desafían las bases de datos, que hoy tienen datos sobre toxicidad relativamente limitados. Los materiales que pertenecen a un mismo compuesto pueden variar considerablemente con respecto a su forma, distribución de tamaño, funcionalización, modificación de superficie, agregación de estados y capacidad de disolución. Debido a esta variabilidad basada en los materiales, hoy en día la ciencia se enfrenta a vacíos de conocimiento sobre el destino y el comportamiento de los materiales de nano ingeniería en los sistemas de prueba y el medio ambiente (Gottschalk, Kost y Nowack, 2013). Por tanto, la nanotecnología y los nanomateriales tienen asociadas impredecibles consecuencias, especialmente en lo que se refiere a sus impactos negativos (Allan et al., 2021), plantean un desafío a la ley (Giusti et al., 2019), pues es necesario regular estas nuevas tecnologías a fin de prevenir violaciones de derechos, y aun así, seguirlas según las metas de desarrollo sostenible, propuestos por la Organización de las Naciones Unidas (ONU) hasta 2030.

\section{La autorregulación y las empresas ciudadanas}

A continuación, se analizan algunas teorías y conceptos de suma importancia para comprender la propuesta de solución (de la pregunta de investigación expuesta), la autorregulación regulada.

La teoría de Ulrich Beck (2018), sobre la "metamorfosis del mundo", aporta un foco importante en la discusión sobre los riesgos. Explicando que en relación con ellos ocurre lo que denomina "desconocimiento fabricado" (Beck, 2018, 137), justifica que nuestro conocimiento de los riesgos globales es altamente dependiente de la ciencia y de los especialistas, siendo que las industrias y especialistas son creadores y evaluadores del riesgo simultáneamente, ocupando una doble posición, y eso mina su posición de poder basada en relaciones de definición de riesgo.

Para intentar romper el círculo vicioso de la ciencia que crea riesgos, se trabajará con la perspectiva del pluralismo jurídico de Gunther Teubner y, a partir de ahí, se estructurarán las bases de la "autorregulación regulada". Engelmann (2017) menciona que, con respecto a la integración de múltiples valores en la toma de decisiones, puede entenderse múltiple como el manejo del pluralismo jurídico a partir de las enseñanzas de Teubner. Aquí es preciso mencionar que el neomonismo precisa estar siempre en busca de normas estatales de inclusión (así como las no escritas), al paso que el pluralismo jurídico presupone la inclusión en un sistema jurídico global (que también incluye el private ordering). Teubner $(2016,101)$ entiende el pluralismo jurídico: "Como la teoría jurídica readecuada a las nuevas fuentes del derecho, llevando en cuenta, así, los procesos espontáneos de formación del derecho en la so- 
ciedad mundial que se revelan independientes de las esferas estatales e interestatales".

La teoría del pluralismo jurídico reconoce el surgimiento de normas jurídicas generadas por nuevos actores de la producción normativa. Según Teubner (Teubner, 2003), en el pluralismo jurídico la producción normativa no está exclusivamente centrada en el Estado, que deja de ser el actor privilegiado o central de la producción normativa y pasa a actuar de otras formas, por ejemplo, como mediador. Así, el pluralismo jurídico de Teubner sostiene la construcción de escenarios jurídicos (como marcos de referencia, árboles de decisión, programas de cumplimiento e integridad), organizados por variados actores de la producción normativa, diferentes a los tradicionales y con motivaciones diversas (políticas, económicas, sociales, tecnológicas), que tienen reconocimiento y eficiencia jurídica.

Cabe mencionar, además, que la decisión sobre el momento más adecuado para estructurar la regulación de los resultados de la innovación es un desafío, y enfrenta dilemas. Uno de esos dilemas fue descrito por David Collingridge, se trata del denominado "Dilema de Collingridge", que puede ser caracterizado a partir de dos problemas opuestos: a) un problema informacional: el caso de las nanotecnologías se encuadra aquí, porque aún no existe suficiente información para estructurar un marco regulatorio; b) un problema de poder: después de que las nanotecnologías estén claramente establecidas, con información suficiente y adecuada para la regulación, habrá dificultades para establecer límites jurídico-legales. El dilema radica en el desafio de elaborar una fórmula regulatoria, que pueda traer seguridad jurídica, pero sin crear obstáculos al desarrollo de la tecnología (Collingridge, 1980).

La regulación tradicional, con la intervención legislativo-estatal, es el modo generalmente esperado por los actores participantes. Sin embargo, el conjunto de las nuevas tecnologías, donde se encuentran las nanotecnologías, acarrea características muy diferentes de las tecnologías convencionales conocidas hasta este momento. En el caso de las nanotecnologías la disrupción es notable, porque las características fisicoquímicas de las nanopartículas y de los nanomateriales hacen que los riesgos sean colocados en niveles y padrones poco conocidos en la actualidad (Nature Nanotechnology, 2019).

En este contexto entra la idea de que, a pesar de no tener una legislación que esté específicamente dirigida a nanopartículas y nanomateriales, pueden extraerse principios del conjunto del ordenamiento jurídico con carácter normativo aplicables a los variados desarrollos de las nanotecnologías, los cuales deberán dialogar con los principios y objetivos internacionales antes mencionados, formando así un cuerpo normativo a ser respetado por todos aquellos que lidian con las construcciones en nanoescala.

Así, para la conformación de este órgano regulador por entidades distintas al Estado, mediante la creación de una autorregulación regulada, es necesario que se entiendan y presenten cuatro conceptos a las empresas e industrias que trabajan con nanotecnologías y nanomateriales, que son: RRI 
(responsible research and innovation), ELSA (ethical, legal and social aspects), la empresa ciudadana y la herramienta de safe by design (seguridad desde el diseño) como parte integrante de estos mecanismos (Soeteman-Hernández et al., 2020).

Todos estos cuatro conceptos están interconectados e interactúan, convirtiéndose en un desafío exponer cada uno de ellos de forma aislada. Así, a continuación, se presentarán aspectos de todos ellos, con el fin de brindar una vista panorámica.

Hace más de veinte años se inició el Programa de Investigación de Implicaciones Éticas, Legales y Sociales (ELSI) del Instituto Nacional de Investigación del Genoma Humano de Estados Unidos, a partir del enfoque para los problemas éticos, legales y sociales que surgen en el contexto de la genética/ genómica (Parker et al., 2019). A partir de estas inquietudes, que deberían orientar la investigación en el área del genoma humano, el contenido de ELSI se extendió a otras áreas del conocimiento. La perspectiva ELSI influyó en la propuesta lanzada por la Unión Europea, años después, de la RRI, en la cual se fijó que apenas se podría considerar efectivamente una investigación e innovación responsables, si estuvieran vinculadas con las nociones de ELSI. En secuencia, surge la herramienta de safe by design, mostrando que la seguridad de los avances científicos debe estudiarse y diseñarse por etapas, poco a poco, dados los diferentes riesgos que podrían derivarse de la exploración humana de la nanoescala. Por tanto, una única estructura de gestión de riesgos para todas las etapas del ciclo de vida de un nanomaterial, no sería eficaz. Por el contrario, para cada etapa del ciclo de vida de un nanomaterial, deben existir estrategias específicas de gestión de riesgos.

El safe by design (SbD) es un concepto bien conocido en la industria. Su aplicación a los nanomateriales fue propuesta por NANoREG por primera vez. Este concepto de SbD está madurando las acciones financiadas por la UE ProSafe y NanoReg2, con el desarrollo de herramientas operativas para apoyar la industria y los reguladores a corto plazo (Gottardo, Huges, Jantunen 2017). El LCA (life cycle assessment) también es un procedimiento bien establecido, pero su aplicación a los nanomateriales se ve obstaculizada actualmente por las incertidumbres metodológicas y la falta de datos, que aún requieren un trabajo científico por resolver.

El objetivo de $\mathrm{SbD}$ es reducir los riesgos en una etapa inicial del proceso de innovación, utilizando el conocimiento científico actual para guiar el diseño de productos con nanomateriales. El SbD propone minimizar las propiedades peligrosas de una sustancia desde las primeras etapas de su desarrollo, de modo que cualquier problema relacionado con el riesgo se aborde en la etapa de proyecto. Además, tiene un enfoque que incorpora los aspectos de seguridad y salud ambiental en una etapa inicial del proceso de innovación de nanomateriales diseñados para garantizar la seguridad de los seres humanos y el medio ambiente (European Comission, 2017). El concepto ha sido utilizado por la industria durante algunos años y está destinado a identificar las 
incertidumbres y los riesgos potenciales lo antes posible durante un proyecto de innovación, así como a identificar las medidas para reducir o eliminar estas incertidumbres y riesgos. No es un concepto independiente, sino diseñado para integrarse en los procesos de innovación actuales.

Según Engelmann (2015), SbD es considerado una herramienta de investigación e innovación responsables (RRI), preocupada con los aspectos éticos, legales y sociales (ELSA), siendo de ELSA, la idea de empresa ciudadana. RRI otorga nuevas responsabilidades, no solo a los científicos, universidades, innovadores y organizaciones, sino también a los responsables por la formulación de políticas y los financiadores de la investigación. La innovación responsable evoca un deber de cuidado colectivo que involucra, en primer lugar, repensar lo que se quiere de la innovación y, luego, cómo puede volverse viable ante la incertidumbre. Reconociendo el poder de la innovación para dar forma a nuestro futuro colectivo, RRI nos desafía, en primer lugar, a preguntarnos qué tipo de futuro queremos que traiga la innovación al mundo (Owen, Macnaghten y Stilgoe, 2012).

La estructura de RRI parece, pensando en algunos de sus puntos de vista y valores hasta ahora presentados, muy prometedora, principalmente cuando se trata de integrar más filosofía de la investigación científica. Por otro lado, la estrecha integración esperada de RRI con proyectos de investigación en curso puede presentar algunos desafíos para estudios más fundamentales de conceptos y supuestos científicos.

Siguiendo las ideas de RRI, surgen aspectos o impactos de ELSA que deben ser tomados en cuenta a la hora de realizar una investigación científica. Una vez más, se busca no bloquear el desarrollo, sino pensar con más seguridad, evitando mayores riesgos, especialmente en lo que respecta a la investigación genómica y nanotecnológica. En la última década se han invertido alrededor de 20 millones de euros en programas de esta categoría. La creación de ELSA ha generado numerosas críticas por centrarse especialmente en aspectos de efectos y resultados (ELSA, 2017; Simeone et al., 2019; Hohendorff, 2018).

Sin embargo, un componente importante del desarrollo responsable de la nanotecnología es la consideración de sus posibles implicaciones éticas, legales y sociales. Para lo cual es útil preguntarse: ¿cómo se introducen en la sociedad la investigación y las aplicaciones de la nanotecnología?, ¿cuán transparentes son las decisiones?, ¿cómo se necesitan políticas sensibles y receptivas y también las percepciones de toda la gama de partes interesadas?, y, ¿cómo se abordarán las cuestiones éticas, legales y sociales para determinar la confianza pública y el futuro de la innovación impulsada por la nanotecnología?

El concepto ELSA se enfoca principalmente en los diversos impactos generados por la ciencia en la sociedad, mientras que la estructura de la RRI sirve para poner mayor énfasis en el proceso de investigación e innovación, apuntando a una mayor implicación y reflexividad —al mismo tiempo que enfatiza la apertura, la transparencia y el diálogo. 
Siguiendo con el concepto ELSA, Hullmann (2008) señala que en nanotecnología, los aspectos éticos, legales y sociales ofrecen una visión importante para el público interesado, ayudando a identificar expectativas e inquietudes y, al mismo tiempo, es importante para la toma de decisiones en las políticas públicas, al corresponder a las necesidades en términos de buena gobernanza de la investigación, incluida la gestión de riesgos. Tales disposiciones de política se enfrentan al desafío de priorizar la investigación financiada con fondos públicos, así como de decidir el futuro de las regulaciones. En este sentido, también destaca que, para dar respuesta a las inquietudes de la sociedad, es fundamental entablar un diálogo sobre los beneficios y riesgos de la nanotecnología, involucrando a gran parte del público y basado en juicio informado.

ELSA, en nanotecnología, comprende una amplia gama de temas relacionados con la investigación, producción y uso de nanotecnología y productos habilitados por ella. Abarcan temas de privacidad, aceptación, salud humana, acceso, responsabilidad, regulación y control, haciendo claramente imprescindible la participación del público consumidor y de la población en general.

La Iniciativa Nacional de Nanotecnología de los Estados Unidos de América (NNI-2017) tiene el compromiso de promover el desarrollo de una comunidad de expertos en temas de ELSA, relacionados con la nanotecnología y construir colaboraciones con diferentes actores, consumidores, ingenieros, filósofos, fabricantes, organizaciones no gubernamentales, reguladores y científicos. Estos grupos de partes interesadas consideran los posibles beneficios y riesgos de los hallazgos en el campo y brindan sus perspectivas sobre las nuevas pautas de investigación. Con las partes interesadas de la industria, la NNI también desarrolla recursos de información para cuestiones éticas y legales relacionadas con la propiedad intelectual y las implicaciones éticas de las patentes basadas en la nanotecnología y los secretos comerciales. Para aumentar la conciencia y la educación de las partes interesadas sobre los temas de ELSA, es necesario difundir el conocimiento apropiado y relevante de estos aspectos a varios actores con diferentes niveles de conocimiento; así, el marco de oportunidades será expandido y nuevos enfoques para las interacciones deliberativas entre grupos de stakeholders (grupos de interés), que son numerosos y diversos, serán desarrollados e implementados. (NNI, 2021).

Actualmente, NNI tiene el centro más grande del mundo con enfoque en los aspectos sociales de la nanotecnología: el Centro para la Nanotecnología en la Sociedad, de la Universidad Estatal de Arizona (ASU, 2021). Una forma de aplicación práctica de los elementos de ELSA se puede encontrar en la concepción renovada de empresa ciudadana, donde la empresa ya no se entiende solo orientada a la obtención de lucro, sino como un grupo humano, que se propone satisfacer las necesidades humanas con calidad (Engellman, 2017).

Como concepto de empresa ciudadana, se puede entender aquella que al actuar asume responsabilidades y no descuida el entorno social o ecológico, limitándose a buscar el máximo beneficio material posible. Y es precisamente este tipo de organización la que actúa inteligentemente porque, com- 
portándose con esta simpatía hacia su entorno, establece una cultura de confianza entre sus integrantes (Cortina, 2005).

Sen (2010: 336) explica que "un código básico de buena conducta en los negocios es un poco como el oxígeno: empezamos a interesarnos por su presencia cuando no está presente". Teubner $(2016,178)$ menciona que "las instancias estatales, como las expectativas normativas, los movimientos sociales y las organizaciones de la sociedad civil, brindan impulsos constitucionales de aprendizaje para la orientación ecológica de las empresas.".

Se verifica, también, la referencia a este código de buena conducta, en la Recomendación de la Comisión al Parlamento Europeo del 7 de febrero de 2008, que trae la idea de un código de conducta para una investigación responsable en el campo de las nanociencias y las nanotecnologías (2008/345/CE).

Esta nueva organización, preocupada por los aspectos éticos, legales y sociales, será fundamental para el desarrollo de una investigación e innovación responsable en el caso de las nanotecnologías, enfocadas en la sostenibilidad en todas sus dimensiones. Como características de esta nueva organización ciudadana, Cortina y Navarro (2015: 167) presentan:

1. Responsabilidad por el futuro La necesidad de una gestión a largo plazo obliga a conciliar el beneficio y el tiempo.

2. Desarrolo de habilidades de comunicación. Toda organización necesita una legitimación social, que se "vende" comunicativamente. El respeto a las normas morales también es un imperativo de las relaciones públicas, ya que es necesario crear un entorno afectivo.

3. Personalización e identificación de personas y empresas. El fracaso del individualismo hace necesario insertar a los individuos en grupos y desarrollar el sentido de pertenencia a ellos. En la competencia entre empresas, los anuncios comerciales no son suficientes para identificar la personalidad de una empresa, sino que se impone el imperativo de la personalización de las empresas. Un claro ejemplo de ello es el patrocinio, que no se ejerce sin ánimo de lucro.

4. En una cultura de comunicación la moral impulsa la creatividad de los especialistas en comunicación y funciona como un medio de diferenciación y personalización de la empresa. En una empresa abierta, la ética es parte del management de 'tercer tipo', basándose en la complejidad de los mercados, no solo el principio de innovación permanente de los productos, sino en la innovación 'moral' de la comunicación.

5. Confianz $a^{2}$ Las imágenes de eficiencias han sido remplazadas por las de confianza entre la empresa y el público. Por ejemplo, en la imagen

\footnotetext{
${ }^{2}$ Sobre la confianza, Luhmann explica que "donde hay confianza hay aumento de posibilidades para la experiencia y la acción, hay un aumento de la complejidad del sistema social y también del número de posibilidades que pueden reconciliarse con su estructura, porque la confianza constituye una forma más efectiva de reducción de la complejidad" (Luhmann, 1996: 14).
} 
de responsabilidad social y ecológica de la empresa con la que se busca establecer un vínculo entre la empresa y el público (énfasis añadido por el autor).

Varias de las características de esta nueva organización están ligadas con la idea de la herramienta SbD, a través de la cual se puede dar el puente que favorecerá la comunicación entre ciencia (productora de innovación) y el derecho. La propia idea de una cultura de la comunicación también se puede ver en la herramienta $\mathrm{SdB}$, así como la innovación moral de la comunicación, y de esta manera, $\mathrm{SbD}$ sería una forma de potenciar el resultado de la comunicación entre diferentes sistemas, como ciencia, derecho, economía, e, incluso, política.

Sobre la confianza, Luhmann $(1996,196)$ señala que una concepción muy compleja y estructurada del mundo no podría establecerse sin una sociedad definitivamente compleja, que, a su vez, no se puede establecer sin confianza - porque la confianza reduce la complejidad social. La confianza es dependiente de los otros mecanismos de reducción, desarrollados paralelamente con esta, por ejemplo, los de la ley, de la organización y, por supuesto, los del lenguaje. Sin embargo, no puede reducirse a ellos.

Los aspectos de responsabilidad social y ambiental de la organización también pasan por la idea de safe en el sentido de colocar un producto más seguro en el mercado. El cuidado con el medio ambiente y con los seres humanos, mediante la adopción de enfoques preventivos y de gestión de riesgos, se puede percibir en la forma en que las organizaciones se comportan con sus consumidores y en la comunidad en la que operan. Las organizaciones exitosas deben tener una visión integral de la gestión de riesgos, que puede considerar cómo protegerse en términos de algunos riesgos, qué riesgos deben explorarse y cómo explotarlos (Damodaran, 2009).

La ética organizacional tiene un papel directo e indirecto en la construcción del desarrollo económico y sus influencias abarcan una amplia variedad de temas, entre ellos, la protección del medio ambiente y la sostenibilidad, el fortalecimiento de los derechos humanos juntamente con el propósito de eliminar la pobreza (Sen, 2007). Castells (en: Cortina, 2007) contribuye al debate sobre la ética organizacional al exponer que las organizaciones también discuten la aplicación de la conciencia ecológica en el proceso empresarial, pasando por todo el proceso de producción.

Este es un problema de conciencia, no de dificultad técnica. Y en ese sentido, es en última instancia una cuestión ética. Antonik $(2016,214)$ explica que la responsabilidad social y ambiental en el área de negocios, es la responsabilidad de la organización con la sociedad y el medio ambiente, además de sus compromisos legales (compliance) y económico-financieros.

Según lo expuesto por Teubner $(2016,175)$ esto significa una "ecologización" de la constitución empresarial, forzada externamente - por parlamentos, gobiernos, sindicatos, movimientos sociales, organizaciones no gubernamen- 
tales, profesiones y medios de comunicación- a cambiar la estructura interna de las empresas, algo que limite las compulsiones de crecimiento excesivo y daño al ambiente. Una constitución corporativa, en este sentido, requeriría que la política corporativa tenga en cuenta las necesidades de su entorno, la naturaleza, la sociedad y los seres humanos, y acompañada de implementaciones internas y control externo.

Así, la responsabilidad social de las organizaciones es, al mismo tiempo, un mejor negocio para la empresa y, además, ayuda en la creación de un mundo mejor y más sostenible, preocupado por la aplicación práctica de los conceptos de RRI y ELSA. Pero, no todo es tan simple y fácil de implementar, especialmente en un mundo donde este no es el concepto de organización que predomina. Así, la idea de organización ciudadana tropieza con innumerables dificultades que obstacularizan su realización.

De esta forma, siendo el objetivo principal una preocupación con la evolución sostenible, la investigación, innovación responsable y con los aspectos éticos, legales y sociales, surge la necesidad de cambios para este modelo de organización ciudadana. Se trata de cambios estructurales que llevan de la jerarquía a la corresponsabilidad, un cambio en la cultura organizacional, la reconfiguración ética del mundo del trabajo, como requisito para hacer frente a los riesgos inciertos y desconocidos que pueden traer las nanotecnologías (Engellman, 2017) y el reposicionamiento del equilibrio social, que no solo representa el equilibrio económico de la organización, sino "también datos sobre el grado de satisfacción que está generando una empresa en la sociedad en la que desarrolla su actividad" (Cortina, 2005: 85-86).

Según Catalán (2008: 92), debido al principio de desarrollo sostenible, las relaciones económicas de una sociedad deberán presentar un comportamiento eco-social, y sus actividades empresariales "necesitan ser administrados como en las civilizaciones helénicas, sin gastar más de lo que ganan, ni retirarse de la naturaleza más de lo que puede ser remplazado". De estos conceptos, han sido propuestas innumerables soluciones para la regulación de las nanotecnologías, que incluyen abordajes voluntarios, cooperativos o en asociación. Pero, a pesar de las grandes ventajas de cada una de ellas, ninguna de las soluciones propuestas para regular las nanotecnologías, hasta el momento, consiguió contemplar dos requisitos importantes: a) la amplia participación de la industria, con la presentación de datos suficientes para auxiliar a los reguladores en relación con la evaluación de riesgos, y, b) las garantías sobre el papel del gobierno en la regulación de tecnologías emergentes que consideren el interés público (ciudadanos) (Abbot, Marchant y Sylvester, 2010; Shandilya et al., 2020; Soeteman-Hernández et al., 2021).

De este modo, es imprescindible la construcción de un nuevo modelo regulatorio, reconocido por el Estado, donde se pueda incluir como característica la efectiva actuación sobre los posibles riesgos ambientales. Siendo necesaria la formulación de políticas públicas e instrumentos de participación de la iniciativa privada, como organizaciones internacionales, agencias regulatorias y 
ONGs, que entiendan la realidad de la crisis ambiental y busquen el enfrentamiento para contemplar la urgente coevolución entre los sistemas de economía y de derecho. Esa coevolución sistémica es posible con la producción de directrices y normas que incorporen en sus formulaciones la necesidad primordial de adaptación de la actuación estatal a la nueva realidad (Weyermüller, 2014).

En el siguiente apartado de este artículo se presenta un ejemplo de producción científica que busca contribuir a la construcción de la denominada autorregulación regulada y brindar orientación a los emprendedores que buscan convertir su empresa o industria en una empresa ciudadana, cumpliendo con los lineamientos que propone este concepto: Cartilla de lineamientos jurídicos para el segmento industrial y comercial que trabaja con nanotecnología, que presenta principios rectores del derecho a los empresarios, cumpliendo el rol de informarles de la existencia de estos, incluso si no existe una legislación que cubra el tema.

\section{Cartilla de lineamientos legales para el segmento industrial y comercial que trabaja con nanotecnología y la autorregulación regulada}

Como se mencionó anteriormente, la nanotecnología trae grandes transformaciones y desafíos a la vida cotidiana, incluidos aquellos que hacen uso de la nanotecnología y los nanomateriales en sus industrias. Por ello, el Grupo de Investigación JusNano, dirigido por el Prof. Dr. Wilson Engelmann, presente en la Universidade do Vale do Rio dos Sinos (UNISINOS) desde 2008, vinculado con el Programa de Posgrado en Derecho (maestría y doctorado) y al Máster Profesional en Derecho de Empresas y Negocios, en el que desarrollan investigaciones relacionadas con los desafíos que enfrenta el derecho debido a la nanotecnología, buscó dar respuesta a las preguntas que surgieron en el entorno empresarial relacionadas con la nanotecnología: ¿cómo avanzar con esa innovación sin retroceder en la protección humana y ambiental?, ¿cómo gestionar una empresa, amparada por la ley, si no existe una legislación específica para las tecnologías "nano"? Así, se elaboró la Cartilla de lineamientos legales para el segmento industrial y comercial que trabaja con nanotecnología con el objetivo de brindar información a los emprendedores, para que crezcan junto con el derecho (Leal, Martins, Hohendorff, Engellman, 2020).

La Cartilla fue el resultado de un amplio y largo trabajo de investigación, con la ayuda de la Fundación para el Apoyo a la Investigación en el Estado de Rio Grande do Sul (FAPERGS) y contiene los principios presentes en nuestra Constitución Federal Brasileña, bien como en las normas técnicas ISO, que puede garantizar la seguridad de la gestión empresarial y del ecosistema, al mismo tiempo que se ocupa de la nanotecnología. Así, es posible mejorar el desarrollo empresarial, ya que los avances y límites serán claros, bajo la guía del derecho y, en consecuencia, habrá protección de los seres vivos y el medio ambiente. 
Se pretende que la Cartilla sea una guía regulatoria para las actividades industriales y comerciales relacionadas con las nanotecnologías ante la falta de una legislación específica. La observación de los principios explicados en la Cartilla puede ayudar en el desarrollo de las nanotecnologías en Brasil, permitiendo una operación más segura y en línea con la ley brasileña (Leal, Martins, Hohendorff, Engellman, 2020).

Estos principios son:

1. Principio de prevención y precaución: el primero busca evitar riesgos al medio ambiente y al ser humano, mediante la adopción de diferentes medidas. $\mathrm{Y}$ el segundo garantiza la protección frente a posibles riesgos que aún no se pueden identificar, debido al precario estado de los conocimientos. Legislación brasileña relacionada con estos principios: Constitución Federal de Brasil (CF). Art. 200, I a VII; 225; caput e incisos IV y V; art. 1, Ley 11.105/2005 (Bioseguridad). Objetivo de la República Federativa del Brasil: promover el bien de todos (CF). Art. 3, iv; Seguridad (CF). Arts. 5, caput y 6, caput; CF. Art. 218, $\S 2$. Política Nacional de Medio Ambiente (Ley 6.368/1981). Política Nacional de Residuos Sólidos (Ley 12.305/2010). Art. 3, X y XI. Gerenciamiento y Gestión Integrada de Residuos Sólidos.

2. Principio de no regresión ambiental: busca el respeto al derecho fundamental al disfrute de un medio ambiente ecológicamente equilibrado para las generaciones presentes y futuras. Legislación brasileña relacionada con este principio: Prevalencia de los Derechos Humanos y de los Tratados Internacionales (CF). Art. 5, § 2 y 3; Art. 4, II; 170, VI; 200, VIII, 225; Política Nacional de los Residuos Sólidos. El destino y disposición final debe ser ambientalmente adecuado (Ley 12.305/2010, Art. 3, VII y VIII); Padrones sostenibles de consumo (Ley 12.305/2010, art. 3, XIII).

3. Principio de transparencia: busca el respeto al derecho de acceso de los ciudadanos, especialmente en su situación de consumidor, a toda la información sobre situaciones que les afectan directamente, especialmente en lo que respecta a su salud y seguridad relacionada también con los productos y servicios que consumen diariamente. Legislación brasileña relacionada con este principio: Derecho/deber de información. Art. 5, XIV, CF. Principio de publicidad administrativa y ambiental (CF). Art. 37, caput y Art. 225, VI. Competencia común de la Unión, Estados y Municipios en proteger el medio ambiente y combatir la contaminación en cualquiera de sus formas (CF). Art. 23, VI. Código de Defensa del Consumidor. Arts. 4, IV; 6, II; 9.

4. Principio de participación del público: está ligado al principio de transparencia, ya que para promover uno se debe promover el otro, buscando respetar el derecho del público a participar en todas las decisiones que involucren la nanotecnología, ya que pueden afectarla 
directamente. Legislación brasileña relacionada con este principio: la misma base descrita en el principio anterior.

5. Principio de la responsabilidad del productor: busca garantizar la existencia de responsables, sean ellos los desarrolladores, los operadores, los fabricantes y/o los vendedores de nanoproductos, por la prevención y la remediación de eventuales daños causados por las nanotecnologias, al ser humano o al medio ambiente. No hay legislación específica en Brasil referente a la responsabilidad del productor con la nanotecnología, pero la legislación relacionada con este principio en otras esferas: Código Civil (responsabilidad por el riesgo del desarrollo). Art. 931; Principio del contaminador pagador; Crímenes Ambientales. Ley 9.605/1998; Art. 225, § 3. Política Nacional de Residuos Sólidos. Art. 3, XVII. Responsabilidad compartida entre fabricantes, importadores, distribuidores, comerciantes, ciudadanos, etcétera.

6. Principio de protección y salud de la población y de los trabajadores: busca la prevención inmediata de exposiciones efectivas o probables durante el proceso de producción o respecto a residuos destinados a disposición o reciclaje, en relación con nanomateriales que presentan riesgos, o incluso aquellos en los que no exista seguridad comprobada. Legislación relacionada con este principio: Defensa del Trabajador (CF). Art. 1, III y IV (dignidad humana; valor social del trabajo y de la libre iniciativa). Defensa del Consumidor (CF). Art. 5, XXXII y 170, V; Arts. 8 hasta 24, Código de Defensa del Consumidor (CDC). Cobertura del riesgo y protección al accidente de trabajo (CF), Art. 7, XXII y Art. 201, § 10. Protección del riesgo a toda la sociedad (CF). Art. 225, $\S 1$, V; art. 170, V. Derecho a la salud (CF). Art. 196.

Por lo tanto, los diferentes medios de producción normativa (estatal y no estatal) no son excluyentes uno del otro, pero merecen pesos distintos una vez que la producción no estatal, bajo el signo de la globalización, puede no ser totalmente compatible con los medios tradicionales de producción normativa (Teubner, 2003: 11).

Respecto a las nanotecnologías y sus riesgos, existe un déficit legislativo que, según Engelmann (2012, 321) hace oportuno, principalmente en la estructura normativa del derecho consolidada a partir de Hans Kelsen, que otros actores de producción jurídica y fuentes de derecho que hasta entonces siempre estuvieran a la sombra del texto de la ley, participen del proceso legislativo. Una de las soluciones para este déficit es la autorregulación regulada, siendo que, para mitigar los efectos negativos sobre la autorregulación, se propone que sea regulada por la participación del Estado, especialmente a partir de una fiscalización del cumplimiento de la parte externa a las organizaciones, que se relaciona con los derechos humanos expresados en los Objetivos de Desarrollo Sostenible y Objetivos de Desarrollo del Milenio de la 
ONU. Por tanto, aunque se hable de autorregulación, existirá un elemento externo con posibilidad de constreñir, y con alguna penalidad para el caso de incumplimiento.

Una alternativa para enfrentar de manera cualitativa los desafíos que aún persisten sobre la autorregulación de los nanomateriales es recurrir a una guía para la gobernanza del riesgo basada en tres niveles (Rodríguez, 2018): 1. Herramientas avanzadas que faciliten la toma de decisiones con base en el riesgo, incluyendo una evaluación de las necesidades de los usuarios en relación con la evaluación, la mitigación y la transferencia de riesgos. 2. Un modelo integrado de comportamiento humano orientado a la toma de decisión sobre riesgos de los nanomateriales. 3. Requisitos jurídico-legales y otros (nanoespecíficos y generales) para garantizar el acuerdo y estimular abordajes proactivos de seguridad. La implementación de tal abordaje deberá facilitar y motivar las buenas prácticas para las partes interesadas, de modo que permita el desarrollo futuro seguro y sostenible de las nanotecnologías (Stone 2018).

\section{Consideraciones finales}

Se puede entender que los cambios y disrupciones provocados por las nuevas tecnologías, en particular la nanotecnología, enmarcados en el contexto de la cuarta revolución industrial, traen grandes desafíos a los distintos sistemas, siendo el derecho muy afectado para mantener su papel dentro de la sociedad para garantizar los derechos. Sin embargo, encuentra obstáculos en el contexto de un crecimiento exponencial y muy rápido de las nuevas tecnologías, haciéndose cada vez más complejo el acompañar estos cambios sociales.

Siendo que el riesgo de las nanotecnologías puede traer consigo importantes violaciones a los derechos humanos y derechos ambientales, especialmente con la falta de legislación en la materia, en este escenario de riesgo como explican Beck y Luhmann, es necesario algún tipo de norma o regulación, y esta no necesariamente debe provenir del Estado, del poder legislativo - lo cual se explica en la teoría del pluralismo jurídico de Teubner, y de cómo es posible crear el derecho a partir de diferentes fuentes, destacando las reglas necesarias para seguir los avances que se dieron con la cuarta revolución industrial.

Por tanto, es a partir de los conceptos y relaciones que se dan entre SbD, RRI, ELSA y empresa ciudadana, que se puede presentar una solución a la pregunta de investigación propuesta, y es a través de estas herramientas que se puede entender y aplicar la autorregulación regulada.

Como ejemplo de la aplicación de esta solución, se presentó la Cartilla de lineamientos legales para el segmento industrial y comercial que trabaja con nanotecnología, iniciativa del Grupo de Investigación JusNano. Con esto, se buscó contribuir para la creación de un reglamento de nanotecnología, presentando la posibilidad de la pluralidad jurídica, de modo que las fuentes del derecho, 
en esta cuarta revolución industrial, no solo estén vinculadas con la legislación producida por el Estado, lo cual es una forma anticuada de abordar las tecnologías disruptivas, puesto que no se consiguen acompañar los cambios de la sociedad y cumplir su papel de manera integral, que debe ser el de proteger los derechos humanos, el derecho al medio ambiente y el derecho al desarrollo sostenible.

\section{Referencias}

Abbot, K. W., Marchant, G. E., Sylvester, D. J. (2010). Transnational regulation of nanotechnology: reality or romanticism? En G. A. Hodge, D. M. Bowman, A. D. Maynard (eds.), International handbook on regulating nanotechnology. Cheltenham: Edward Elgar.

Allan, J. et al. (2021). Regulatory landscape of nanotechnology and nanoplastics from a global perspective. Regulatory Toxicology and Pharmacology, 122(104885). https://www.sciencedirect.com/science/article/pii/S0273230021000258

Antonik, L. R. (2016). Compliance, ética, responsabilidade social e empresarial: uma visão prática. Rio de Janeiro: Alta Books.

ASU. Arizona State (2021). University. The Center for Nanotechnology in Society. (2021). http://cns.asu.edu

Beck, U. (2003). Un mondo a rischio. Traducción de Laura Castoldi. Torino: Giulio Einaudi.

Beck, U. (2010). Sociedade de risco: rumo a uma outra modernidade. Traducción de Sebastião Nascimento. São Paulo: Ed. 34.

Beck, U. (2015). Sociedade de risco mundial: em busca da segurança perdida. Traducción de Marian Toldy y Teresa Toldy. 1a ed. Lisboa: Edições 70, 2015. Documento disponible para Kindle.

Beck, U. (2018). A metamorfose do mundo: novos conceitos para uma nova realidade. Revista de Direito Econômico e Socioambiental, 9(3), São Paulo. https://doi. org/10.7213/rev.dir.econ.soc.v9i3.24815

Carvalho, D. W. de, Damacena, F. D. L. (2013). Direito dos desastres. Porto Alegre: Livraria do Advogado.

Castells, M. (2007). Para além da caridade: responsabilidade social no interesse da empresa na nova economia. En Cortina, Adela (org.). Construir confiança: ética da empresa na sociedade da informação e das comunicações. Traducción de Alda da Anunciação Machado. São Paulo: Edições Loyola.

Catalan, M. J. (2008). Proteção constitucional do meio ambiente e seus mecanismos de tutela. São Paulo: Método.

Collingride, D. (1980). The social control of technology. Nueva York: St. Martin's.

Cortina, A. (2005). Cidadãos do mundo: para uma teoria da cidadania. Traducción de Silvana Cobucci Leite. São Paulo: Loyla.

Cortina, A., Navarro, E. M. (2015). Ética. São Paulo: Edições Loyola.

Damodaran, A. (2009). Gestão estratégica do risco: uma referência para a tomada de riscos empresariais. Porto Alegre: Bookman. 
Dana, D. A. (2012). The nanotechnology challenge: creating legal institutions for uncertain risks. Nueva York: Ed. Cambridge University Press.

Durán, N., Mattoso, L. H. C., Morais, P. C. de. (2006). Nanotecnologia: introdução, preparação e caracterização de nanomateriais e exemplos de aplicação. São Paulo: Artliber.

ELSA. (2017). What is ELSA research - in a Norwegian context? https://www.ntnu. no/blogger/elsa/whatiselsanorway/what-is-elsa-research-in-a-norwegiancontext

Engelmann, W. (2012). O diálogo entre as fontes do direito e a gestão do risco empresarial gerado pelas nanotecnologias: construindo as bases à juridicização do risco. En L. L. Streck; L. S. Rocha, W. Engelmann. (orgs.). Constituição, sistemas sociais e hermenêutica: anuário do Programa de Pós-Graduação em Direito da unisinos: mestrado e doutorado: no. 9. Porto Alegre-São Leopoldo: Livraria do Advogado/Unisinos.

Engelmann, W. (2015). As nanotecnologias como um exemplo de inovação e os reflexos jurídicos no cenário da pesquisa e inovação responsáveis (responsible research and innovation) e das implicações éticas, legais e sociais (ethical, legal and social implications). En Streck, L. L., Rocha, L. S., Engelmann, W. (org.). Constituição, sistemas sociais e hermenêutica: anuário do Programa de Pós-Graduação em Direito da UNISINOS: mestrado e doutorado: $n$. 12. Porto Alegre: Livraria do Advogado; São Leopoldo: Ed. Unisinos.

Engelmann, W. (2017). O pluralismo das fontes do direito como uma alternativa para a estruturação jurídica dos avanços gerados a partir da escala manométrica. En Streck, L. L., Rocha, L. S., Engelmann, W. (org.). Constituição, sistemas sociais e hermenêutica: anuário do Programa de Pós-Graduação em Direito da UNISINOS: mestrado e doutorado: $n$. 13. Porto Alegre: Livraria do Advogado; São Leopoldo: Ed. Unisinos.

Engelmann, W., Flores, A. S., Weyermüller, A. R. (2010). Nanotecnologias, marcos regulatórios e direito ambiental. 1. ed. Curitiba: Honoris Causa.

European Comission. (2017). Science for environment policy. Assessing the environmental safety of manufactured nanomaterials: in-depth report 14. Bristol. http://ec.europa.eu/environment/integration/research/newsalert/pdf/assessing_environmental_safety_nanomaterials_IR14_en.pdf

Foladori, Guillermo et al. 2015. Nanotecnologías en América Latina: trabajo y regulación. Zacatecas, México: Ed. Universidad Autónoma de Zacatecas.

Giusti, A. et al. (2019). Nanomaterial grouping: existing approaches and future recommendations. NanoImpact, 16(100182). https://www.sciencedirect.com/science/ article/abs/pii/S2452074819300916

Gottardo, S., Huges, C., Jantunen, P. (ed.). (2017). NANoREG framework for the safety assessment of nanomaterials. JRC Science for Policy Report, Brussels. http://publications.jrc.ec.europa.eu/repository/bitstream/JRC105651/kjna28550enn.pdf

Gottschalk, Fadri; Kost, Elias; Nowack, Bernd. (2013). Engineered nanomaterials in water and soils: a risk quantification based on probabilistic exposure and effect modeling. Environmental Toxicology and Chemistry, v. 32, no. 6: 1278-1287. 
https://www.ncbi.nlm.nih.gov/pubmed/23418073

Guivant, J. S. (2001). A teoria da sociedade de risco de Ulrich Beck: entre o diagnóstico e a profecia. Estudos Sociedade e Agricultura, 16, abr. Rio de Janeiro. http://r1.ufrrj.br/ esa/V2/ojs/index.php/esa/article/download/188/184

Hansen, S. F., Baun, A., Alstrup-Jensen, K. (2011). NanoRiskCat - A conceptual decision support tool for nanomaterials. Copenhagen: The Danish Environmental Protection Agency, (Environmental project, n. 1372). https://www2.mst.dk/ udgiv/publications/2011/12/ 978-87-92779-11-3.pdf

Hohendorff, R. v., Engelmann, W. (2014). Nanotecnologias aplicadas aos agroquímicos no Brasil: a gestão do risco a partir do diálogo entre as fontes do direito. Curitiba: Juruá.

Hohendorff, R. v. (2018). A contribuição do safe by design na estruturação autorregulatória da gestão dos riscos nanotecnológicos: lidando com a improbabilidade da comunicação inter-sistêmica entre o direito e a ciência em busca de mecanismos para concretar os objetivos de sustentabilidade do milênio. Tesis de doctorado. Programa de Pós-Graduação em Direito Público. 480 p. http://www.repositorio.jesuita. org.br/handle/UNISINOS/7055

Hullmann, A. (2008). European activities in the field of ethical, legal and social aspects (ELSA) and governance of nanotechnology. Nano and Converging Sciences and Technologies, Luxembourg, oct. http://cordis.europa.eu/ pub/nanotechnology/ docs/elsa_governance_nano.pdf

Kühnel, D. et al. (2014). Environmental impacts of nanomaterials: providing comprehensive information on exposure, transport and ecotoxicity - the project DaNa2.0. Environmental Sciences Europe, 26(21). http://www.enveurope.com/ content/26/1/21

Leal, D. W., Martins, P. S., Hohendorff, R. v.; Engelmann, W. (2020). Orientações Jurídicas para o Segmento Industrial e Comercial que Trabalha com Nanotecnologias: construindo estruturas normativas a partir dos princípios. São Leopoldo: Karywa.

Leite, J. R. M., Ayala, P. de A. (2010). Dano ambiental: do individual ao coletivo extrapatrimonial. Teoria e prática. 3. ed. rev., atual. e ampl. São Paulo: Revista dos Tribunais.

Luhmann, N. (1996). Confianza. Introducción de Dario Rodríguez Mansilla. Barcelona: Anthropos; México: Ed. Universidad Iberoamaericana; Santiago do Chile: Instituto de Sociología: Ed. Pontifícia Universidad Católica de Chile.

Luhmann, N. (2006). Sociologia del riesgo. Tradução de Silvia Pappe, Brunhilde Erker e Luis Felipe Segura. México: Ed. Universidad Iberoamericana.

Maynard, A. (2016). Não falamos mais sobre riscos da nanotecnologia, mas isso não significa que eles desapareceram. Tecnologias Emergentes, Sociedade e Desenvolvimento, http://nanotecnologiasociedade.weebly.com/blog/nao-falamos-maissobre-riscos-da-nanotecnologia-mas-isso-nao-significa-que-desaparecerampor-andrew-maynard

Nature Nanotechnology. n. d. Nanomaterials Definition Matters. (Consultado en 2019). www.nature.com/naturenanotechnology

National Nanotechnology Initiative (NNI). (2021). Ethical, legal and societal issues. 
http://www.nano.gov/you/ethical-legal-issues

Organisation for Economic Co-Operation and Development (OECD). (2017). Consumer and environmental exposure to manufactured nanomaterials. Information used to characterize exposures: analysis of a survey. (Series on the safety of manufactured nanomaterials, n. 84). París: ENV/JM/MONO.

Ost, F. (1999). O tempo do direito. Traducción de Maria Fermanda Oliveira. Lisboa: Instituto Piaget.

Owen, R., Macnaghten, P., Stilgoe, J. (2012). Responsible research and innovation: from science in society to science for society, with society. Science and Public Policy, 39(6), dec. Londres. https://academic.oup.com/spp/article/39/6/751/1620724

Parker, L. S. et al. (2019). Normative and conceptual ELSI research: what it is, and why it's important. Genetics in medicine. Official Journal of the American College of Medical Genetics, 21(2). https://pubmed.ncbi.nlm.nih.gov/29970926/

Pavlicek, A. et al. (2021). A European nano-registry as a reliable database for quantitative risc assessment of nanomaterials? A comparison of national approaches. NanoImpact, 21(100276). https://www.sciencedirect.com/science/article/pii/ S2452074820300707

Rocha, L. S., MartinI, S. R. (2016). Teoria e prática dos sistemas sociais e direito. Porto Alegre: Livraria do Advogado.

Rodríguez, H. (2018). Nanotechnology and risk governance in the European Union: the constitution of safety in highly promoted and contested innovationn areas. NanoEthics 12(1).

Schwab, K. (2016). A quarta revolução industrial. Traducción de Daniel Moreira Miranda. São Paulo: Edipro.

Sen, A. (2010). Desenvolvimento como liberdade. Traducción de Laura Teixeira Motta. São Paulo: Companhia das Letras.

Sen, A. (2007). Ética de empresa e desenvolvimento econômico. En Cortina, A. (org.), Construir confiança: ética da empresa na sociedade da informação e das comunicações. São Paulo: Edições Loyola.

Shandilya, N. et al. (2020). Perspective on a risk-based roadmap towards the implementation of the safe innovation approach for industry. NanoImpact, 20(100258). https://www.sciencedirect.com/science/article/abs/pii/S2452074820300525

Simeone, F. C., Blosi, M., Ortelli, S., Costa, A. L. (2019). Assessing occupational risk in designs of production processes of nano-materials. NanoImpact, 14(100149). https://www.sciencedirect.com/science/article/pii/S2452074818301794

Soeteman-Hernández, L. G. et al. (2020). Challenges of implementing nano-specific safety and safe-by-design principles in academia. NanoImpact, 19(100243). https://www.sciencedirect.com/science/article/pii/S2452074820300379

Soeteman-Hernández, L. G. et al. (2021). Modernizing innovation governance to meet policy ambitions through trusted environments. NanoImpact, 21(100301). https://www.sciencedirect.com/science/article/pii/S2452074821000100

Stone, V. et al. (2018). The essential elements of a risk governance framework for current and future nanotechnologies. Risk Analysis, 38(7).

Teubner, G. (2003). A Bukowina global sobre a emergência de um pluralismo jurídico transna- 
cional.http://livrozilla.com/doc/1623775/a-bukowina-global-sobre-a-emerg\%c3\%a Ancia-deum-pluralismo

Teubner, G. (2016). Fragmentos constitucionais: constitucionalismo social na globalização. Coordinación de Marcelo Neves et al. y revisión técnica de Pedro Ribeiro y Ricado Campos. São Paulo: Saraiva.

Weyermüller, A. R. (2014). O estado ambiental da adaptação: um novo paradigma. Revista da Ajuris, 41(134): 29-56. http://www.ajuris.org.br/ojs2/index.php/ revajuris/article/view/194/130 